\title{
An Improved Multi-Bit Threshold Flipping LDPC Decoding Algorithm
}

\author{
Lina Zhang, Zhihui Ye, and Qi Feng
}

\begin{abstract}
This paper considers error probability of much larger absolute value symbols about reliability rate based weighted bit-flipping (RRWBF) algorithm of low density parity check code (LDPC). RRWBF owns better performance and effective practicability, compared with weighted bit flipping algorithm (WBF). This paper proposes a modified version of this algorithm, multi-bit-threshold rate based weighted bit flipping about LDPC decoding algorithm (MTRWBF). The scheme solved for RRWBF and its modified algorithms only consider flipping greater weight and slurs the importance of the absolute value of symbols. This algorithm turns a plurality of bits every time, which overcomes shortcomings that IRRWBF decoding convergence speed is too slow. Simulation results showed that the improved version greatly fastens simulation speed, observable reduces the decoding iterations and improves decoding performance. Compared with RRWBF, the error ratio of the MTRWBF with $5 \mathrm{~dB}$ is improved from 10-3 to $10-4$ as proved by simulation results.
\end{abstract}

Index Terms-Low density parity check code (LDPC), iterative decoding, weighted bit-flipping (WBF), reliability rate.

\section{INTRODUCTION}

LOW, density parity check (LDPC) code was first proposed in 1962 by Gallager [1], and has drawn significant attention, mainly because of their extraordinary performance and near to the Shannon limit by using iterative decoders. LDPC code as the very long code length has the characteristics of resistance to burst error. LDPC code has a more systematically constructive method using deterministic such as Euclidean geometric low density parity check (EGLDPC) codes, which is constructed deterministically using the points and lines of a Euclidean geometry [2]. Furthermore, their encoding can be easily implemented, which can be serial iterative decoding or parallel algorithm. The parallel algorithm can be efficiently implemented with linear shift registers. In addition, with the further study of LDPC, it is very good candidates for use in applications like the $4 \mathrm{G}$ mobile communications which need fast decoders and low bit-error rates (BERs).

LDPC codes can be decoded with many encoding methods, which are classified as some classes according to the construction method. Among them, the construction of

Manuscript received November 9, 2013; revised March 20, 2014. This work was supported in part by the National Natural Science Foundation of China under Grant 60932002, in part by Jiangsu Province Science and Technology Support Programme (Industry) under Grant BE 2012155, and in part by Special Research Foundation of Marine Public Service Sector under Grant 201205035.

The authors are with the School of Electronic Science and Engineering, Nanjing University, Nanjing 210023, China (e-mail: zln654321@163.com, yezh@nju.edu.cn,xiaoyehouzi@126.com).
Gallager shows good error performance with different decoding codes. The LDPC that construction of Gallager has uniform weight in every row and column that results in regular encoder and easy decoding implementations. The proper construction of LDPC coders can get large minimum distance that prevents the occurrence of error floor, which pondered a serious disadvantage of well-performing random LDPC codes compared to the arithmetic code.

LDPC codes can be decoded using soft-decision decoding algorithm such as belief propagation (BP), hard-decision decoding algorithm such as bit-flipping (BF), and hybrid decoding methods, which develops the soft BF to take advantage of both BP and BF algorithms [3], [4]. Soft decoding algorithms owns well performance while require much higher decoding complexity like BP decoding algorithm and its improved algorithm such as Sum-Product (SP) [5], the minimum decoding algorithm (Min-Sum, MS) [6], and improved minimum decoding algorithm (Modified min-sum, MMS) [7]. SP algorithm is not easy to implement in hardware and requires large memory and high interconnection complexity resulting from high variable and column degrees. Instead, hard-decision decoding algorithm and hybrid decoding methods balance between hardware implementation and complexity, although the bit error rate performance of the $\mathrm{BF}$ is inferior to that of $\mathrm{BP}$ algorithm. A hybrid decoding method follows the basic form of BP and $\mathrm{BF}$ algorithms to reduce the complexity. A hard-decision decoding algorithm hybrid decoding method [8] is the foundation of all kinds of hybrid decoding method and many variants have been developed. Therefore it also has widespread application. In recent few years, a variety of BF hard decision algorithms based on improved algorithm weighted bit-flipping decoding (weighted bit-flipping, WBF) algorithm [9], the reliability ratio weighted bit-flipping algorithm (reliability rate based weighted-bit-flipping, RRWBF) [10], and improved reliability ratio weighted bitflipping algorithm (improved reliability rate based weightedbit-flipping, IRRWBF) algorithm [11] maintains the low complexity and well decoding performance to make it the further improvement and more practical. In the present paper, a novel modification of IRRWBF is proposed which is called multi-bit threshold LDPC weighted ratio flipping decoding algorithm (multi-bit-threshold rate based weighted bit-flipping, MTRWBF). With a slight increasing in computing complexity, the proposed scheme ignores that existing bit-flipping-based RRWBF only consider flipping greater weight and the influence of the absolute value. It fastens decoding convergence speed and has a better decoding performance.

The article is organized as follows. Section II briefly shows the reviews traditional WBF, RRWBF and IRRWBF decoders. MTRWBF algorithm and relative parameters are 
presented in Section III. In Section IV, decoding complexity comparison is discussed. We provide simulation results and analysis in Section V. Finally, the conclusion is in Section VI.

\section{ANALYSIS OF LDPC DECODING AlgorithM}

\section{A. WBF Algorithm}

Simulation environment: The communication channel is AWGN, through BPSK modulation, $\boldsymbol{H}_{m \times n}$ represents $m \times n$ binary parity-check matrix of the LDPC codes, $\boldsymbol{c}=\left[c_{1}, c_{2}, \ldots\right.$ ,$c_{n}$ ] represents transmitted sequence, the received sequence is $\boldsymbol{y}=\left[y_{1}, y_{2}, \ldots, y_{n}\right], \boldsymbol{b}=\left[b_{1}, b_{2}, \ldots, b_{n}\right]$ is the hard decision. Denote $\boldsymbol{M}(n)=\left\{m: \boldsymbol{H}_{m n}=1\right\}$ is the set that participate in check $m$ and $\boldsymbol{M}(n)=\left\{m: \boldsymbol{H}_{m n}=1\right\}$ indicates the bit $n$ involves the set of checks. Set $N(m)=\left\{n: H_{m n}=1\right\}$ represents the $m$-th parity equations involved in the bit set [12], $|y|_{\min -m}$ represents parity component of metrics $s_{m}$.

$\mathrm{BF}$ algorithm can be seen as the simplest algorithm for weighted bit flipping, which the weight of the bit only contains information on its corresponding check equations. In recent years, emerges many improved the weighted bitflipping algorithm and varies from the calculation of weights, but the ultimate goal still want fast and accurately locate the bit error. WBF use some reliability information, which can obtain better performance than $\mathrm{BF}$, especially the larger weight, such as finite geometric codes [13]. Original WBF algorithm, we separate the decoding algorithm into four steps: initialization, check node, variable node, and decision steps. The operations performed at the specific decoding step can be calculated as follows [7]

$$
\text { Initialization: } j=1,2, \ldots, M,|y|_{\min -m}=\min _{n: n \in N(m)}\left|y_{n}\right|
$$$$
\text { Check node: } m=1,2, \ldots, M, s_{m}=\sum_{n=1}^{N} b_{n} \boldsymbol{H}_{n m} \text {; }
$$

Variable node: $n=1,2, \ldots, N$,

$$
E_{n}=\sum_{m \in \boldsymbol{M}(n)}\left(2 s_{m}-1\right)|y|_{\min -m}
$$

Decision: Flip the bit $b_{k}$ for $E_{k}=\max \left(E_{n}\right)$.

Repeat between check nodes to decision step until all of the check equations satisfies or reaches the set maximum number of iterations. Otherwise decoding fails.

\section{B. Reliability Rate Weighted Bit-Flipping Algorithm}

The destruction is due to the smallest absolute value symbols in WBF, but if a parity equation is wrong, all the relevant symbols have the possibility of error in this equation. The only difference is that the absolute value of the error is smaller, the greater the likelihood of error symbols. In fact, large absolute value means error possibilities and should not be ignored. For the obvious shortcomings of WBF, RRWBF algorithm introduces a new criterion called reliability ratio (RR), where $\left|y_{m}^{\max }\right|$ is the maximum absolute value symbols in the equations values in the $m$-th parity. $\beta$ is a normalization factor to ensure $\sum_{n \in N(m)} R_{m n}=1$. The specific steps are organized as follows [2]

Initialization: $R_{m n}=\beta \frac{\left|y_{n}\right|}{\left|y_{m}^{\max }\right|}, y_{m}^{\max }=\max _{n \in N(m)}\left|y_{n}\right| ;$

Check node: $s_{m}=\sum_{n=1}^{N} b_{n} \boldsymbol{H}_{n m}$;

Variable node: $E_{n}=\sum_{m \in M(n)}\left(2 s_{m}-1\right) / R_{m n}$;

Decision: Flip the bit $b_{k}$ for $E_{k}=\max \left(E_{n}\right)$.

Repeat between check nodes to decision step until all of the check equations satisfies or reaches the set maximum number of iterations. Otherwise decoding fails. It can be concluded that except for the calculation of the variable node and initialization information, the process is the same in Section 2A.

\section{Improved RRWBF}

According to the normalization factor

$$
\sum_{n \in N(m)} R_{m n}=1
$$

such that

$$
\sum_{n \in N(m)}\left[\beta\left(\left|y_{N}\right| /\left|y_{m}^{\max }\right|\right)\right]=1
$$

Taking into account $\beta$ is the independent to $\left|y_{m}^{\max }\right|$ that

$$
\sum_{n \in N(m)}\left|y_{n}\right|=\left|y_{m}^{\max }\right| / \beta
$$

$T_{m}=\sum_{n \in N(m)}\left|y_{n}\right|$, the weights can be rewritten as $E_{n}=\frac{1}{\left|y_{n}\right|} \sum_{m \in M(n)}\left(2 s_{m}-1\right) T_{m}$, which is more concise and less computation than RRWBF. In addition, the decoding principle can be more clearly seen. $\left|y_{n}\right| / T_{m}$ represents the proportion of absolute value symbol. The specific decoding steps are organized as follows [9]

$$
\begin{aligned}
& \text { Initialization: } T_{m}=\sum_{n \in N(m)}\left|y_{n}\right| \\
& \text { Check node: } s_{m}=\sum_{n=1}^{N} b_{n} \boldsymbol{H}_{n m}
\end{aligned}
$$

Variable node: $E_{n}=\frac{1}{\left|y_{n}\right|} \sum_{m \in M(n)}\left(2 s_{m}-1\right) / T_{m}$;

Decision: Flip the bit $b_{k}$ for $E_{k}=\max \left(E_{n}\right)$.

Repeat between check nodes to decision step until all of the check equations satisfies or reaches the set maximum number of iterations. Otherwise decoding fails. Similarly, except for the calculation of the variable node and initialization information, the process is the same in Sections $2 \mathrm{~A}$ and $2 \mathrm{~B}$. 


\section{Multi-Bit Threshold LDPC RATio DeCoding ALGORITHM}

For the AWGN channel, the relationship of the likelihood ratio of the absolute value about the each output $y_{n}$ of the received sequence $\boldsymbol{y}$ for associated with the hard-decision $b$ is the following

$$
\varsigma=\mid \ln \left(p\left(y_{n} \mid c_{n}=1\right) / p\left(y_{n} \mid c_{n}=0\right)|/| y_{n} \mid\right.
$$

where $\varsigma$ is proportional factor.

From (4) shows that $\left|y_{n}\right|$ is the greater and the reliability of the hard decision is the higher. Therefore, the probability of error is smaller. For $\boldsymbol{y}=\left[y_{1}, y_{2}, \ldots, y_{n}\right]$, using absolute value $\left|y_{n}\right|$ as the reliability measurement of a hard decision. However, in Section 2B and Section 2C only considered the ratio of absolute value while the symbol information is not reflected [14]. As long as these two algorithms have the same proportions, the corresponding algorithms are equivalent to treatment and ignore the absolute value of information.

Furthermore, in the decoding process of the abovementioned WBF, RRWBF, IRRWBF, every time only one bits is flipped according to its bit flipping rule that the bits corresponding to the maximum. The decoding speed is too slow, which results in the decoding speed inefficient. This article combines multi-bit flipping algorithm LCMBF [15] and multi GDBF algorithm[11], which permits multiple bit flipping during each iteration of a decoding process, and two shortcomings listed above of RRWBF [16], and proposes a multi-bit threshold ratio LDPC weighted bit flipping decoding algorithm LMRWBF. MTRWBF exhibits faster convergence than the those in above-mentioned WBF and other variants. Therefore, MTRWBF is a lowcomplexity high-weigh LDPC decoding method.

\section{A. MTRWBF Algorithm}

Denote set $\boldsymbol{Q}$ as the absolute value of the discriminate, and $\delta$ is the threshold used to determine the magnitude of the smaller symbols.

Initialization: $\boldsymbol{Q}=\left\{b_{n} \leq \delta\right\}, T_{m}=\sum_{n \in N(m)}\left|y_{n}\right|$ Check node: $s_{m}=\sum_{n=1}^{N} b_{n} \boldsymbol{H}_{n m}$;

Variable node: $E_{n}=\frac{1}{\left|y_{n}\right|} \sum_{m \in M(n)}\left(2 s_{m}-1\right) / T_{m}$;

Decision: $E_{k \max }=\max \left(E_{n}\right)$, flipping the bit satisfies $b_{n} \in\left\{x \mid \theta E_{n \text { max }}<x \leq E_{n \max }\right\}$ and $b_{n} \in\left\{x \mid x<y_{k}\right\}$.

Repeat between check nodes to decision step until all of the check equations satisfies or reaches the set maximum number of iterations. Otherwise decoding fails.

\section{B. Select Parameters $\gamma$ and $\delta$}

For LMRWBF algorithm, the size of $\gamma$ affects the frequency of bit-flipping directly, leading to bits several times flipping and suffers from the heavy oscillation behavior of a decoder, which is not easy to control. Selecting the different parameter $\theta$ influences proper decoding. It is necessary to choose the optimal value. Fig. 1 shows the optimal value is 0.25 when the maximum number of iterations is set to 20 .

On the other hand, $\delta$ is a real value, which can be determined by simulation. By choosing proper $\delta$ can not only make the improved algorithm achieve better decoding performance, but also make the decoding performance stability. Moreover, $\delta$ is influenced by $\gamma$. Fig. 2 describes BER performance with different $\delta$. It can be shown that the decoder can obtain relatively stable and moderate decoding performance when $\delta$ is set to 0.4 .

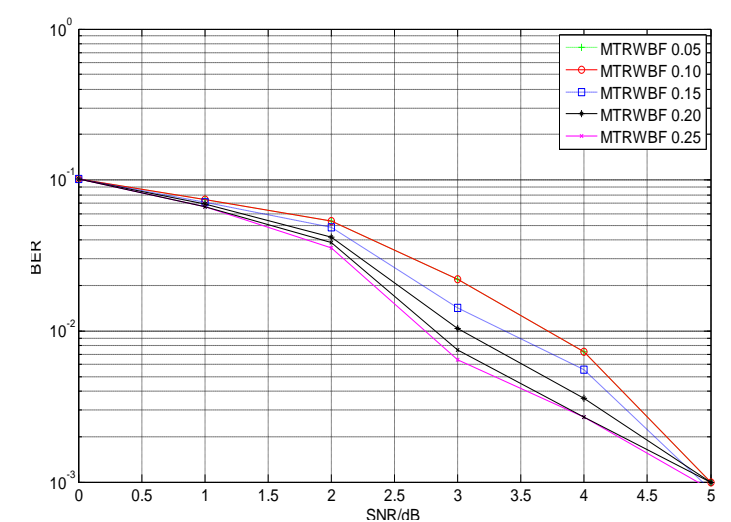

Fig. 1. Improved algorithm performance when selecting different $\gamma$.

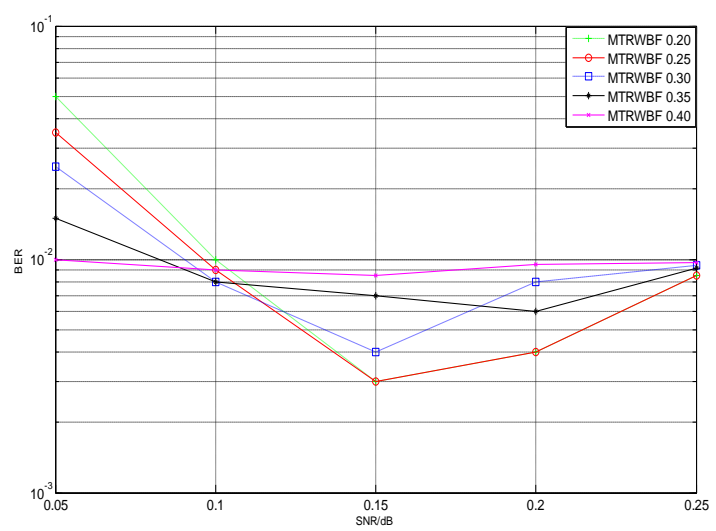

Fig. 2. BER performance with different $\delta$.

\section{ANALYSIS OF COMPUTATIONAL COMPLEXITY}

Consider decoding complexity comparison in all list decoding algorithms. Compared with the logical operation, real number operation is much more complex. Hence, the complexity of the decoding algorithm is mainly determined by the amount of the operation of real number. In the presented paper, we mainly concentrated on the comparison of regular LDPC codes in all decoding algorithms. Assuming the rate of LDPC code is $1 / 2$, we calculate the complexity of WBF, RRWBF, IRRWBF and LMRWBF. $W_{r}$ is the number of nonzero entries in each row of parity check matrix, $W_{c}$ is the number of nonzero entries in each column of parity check matrix. The complexity of different algorithms is shown in Table I.

Consider the proposed algorithm as well as several other algorithms, each iteration requires selecting multiple bits to be flipped, and the computational increase linearly. In the decision step, other algorithms computational complexity is more $N-1$ than other algorithms. In the initialization step, 
LMRWBF and IRRWBF apparently have less absolute computational complexity. All above mentioned algorithms in Table I, have near to complexity in the check node and variable node steps. In general, WBF, RRWBF and IRRWBF are the complexity of $O(N)$, respectively. However, the proposed LMRWBF remains the complexity of $O(N)$ while it has much more performance.

TABLE I: COMPARISON OF COMPUTATIONAL COMPLEXITY

\begin{tabular}{ccccc}
\hline \hline Algorithm & Initialization & Check node & Variable node & Decision \\
\hline WBF & $M\left(W_{r}-1\right)$ & $W_{c}\left(W_{r}-1\right)$ & $W_{r}\left(W_{c}-1\right)$ & $N-1$ \\
RRWBF & $M\left(W_{r}-1\right)$ & $W_{c}\left(W_{r}-1\right)$ & $W_{r}\left(W_{c}-1\right)$ & $N-1$ \\
& $W_{c} W_{r}$ & & $W_{r}\left(W_{c}-1\right)$ & $N-1$ \\
IRRWBF & $W_{c}\left(W_{r}-1\right)$ & $W_{c}\left(W_{r}-1\right)$ & $W_{c}$ & {$[(N-1)$,} \\
& $N$ & & $W_{r}\left(W_{c}-1\right)$ & {$\left[\left(W_{r}-1\right)\right.$} \\
LMRWBF & $W_{c}\left(W_{r}-1\right)$ & $W_{c}$ & $2(N-1)]$ \\
\hline \hline
\end{tabular}

Adder Operator: Initialization $W_{c}\left(W_{r}-1\right), W_{r}\left(W_{c}-1\right)$

Multiplication Operator: Check node $W_{c}\left(W_{r}-1\right)$

Divider Operator: $W_{c}, W_{c} W_{r}$

Absolute Operator: $M\left(W_{r}-1\right)$

Comparison Operator: $N-1, N,[N-1,2(N-1)]$

\section{Simulation Results}

LDPC codes simulation platform based on Simulink 7.10.0 environment makes use of $S$-function model which provides a function code and simulink interface function to complete decoding algorithm implementation. For LDPC codes decoding and established a Matlab/Simulink 7.0 simulation environment for system simulation platform, the specific simulation conditions are as follows.

1) Gallager structure which generates a random parity check matrix $\boldsymbol{H}, K=204, N=408, M=204$, code rate $R=0.5$, the weight of column is 3 .

2) BPSK modulation, assuming the transmission channel is the additive white Gaussian noise (AWGN).

3) Each SNR point samples 50 error code.

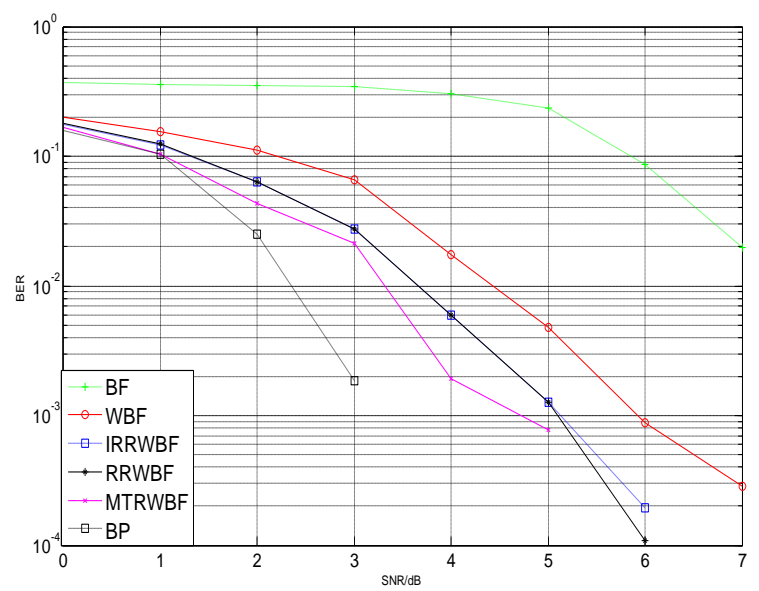

Fig. 3. Comparison of performance for maximum number of 10 iterations.

The comparison of performance in the proposed MTRWBF algorithm and WBF, RRWBF, IRRWBF with the same iterations shows in Fig. 3 and Fig. 4. BP algorithm has the best performance and faster convergence speed while requires much more simulation time because of its high complexity. BF algorithm is seen as the simplest algorithm, and shows the worse performance although it is few complexity and easy hardware implementation. MTRWBF has a good performance between BF and BP. Moreover, it is better decoding performance and faster convergence speed than WBF, RRWBF and IRRWBF. When the number of iterations increases, MTRWBF decoding performance is much closer to BP decoding algorithm. It can be shown in Fig. 5 that compared with several other decoding algorithms, the proposed MTRWBF algorithm requires fewer iterations, which consequently lead to lower complexity. As shown in Fig. 4, compared with RRWBF, the error ratio of the MTRWBF with $5 \mathrm{~dB}$ is improved from $10^{-3}$ to $10^{-4}$.

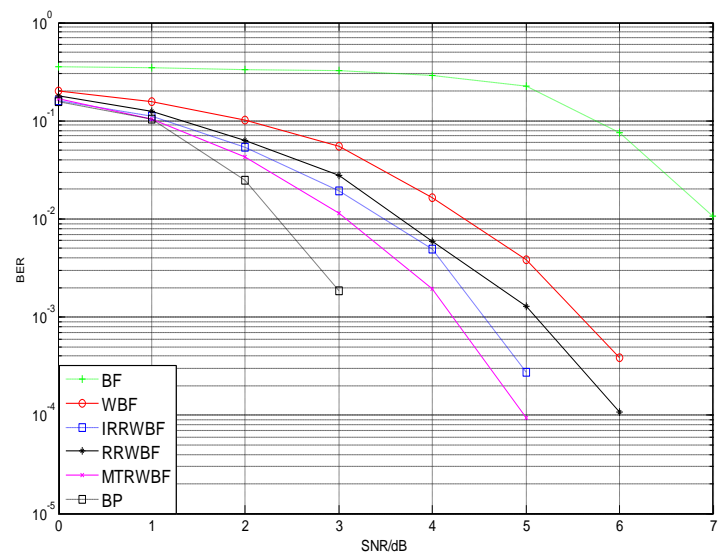

Fig. 4. Comparison of performance for maximum number of 20 iterations.

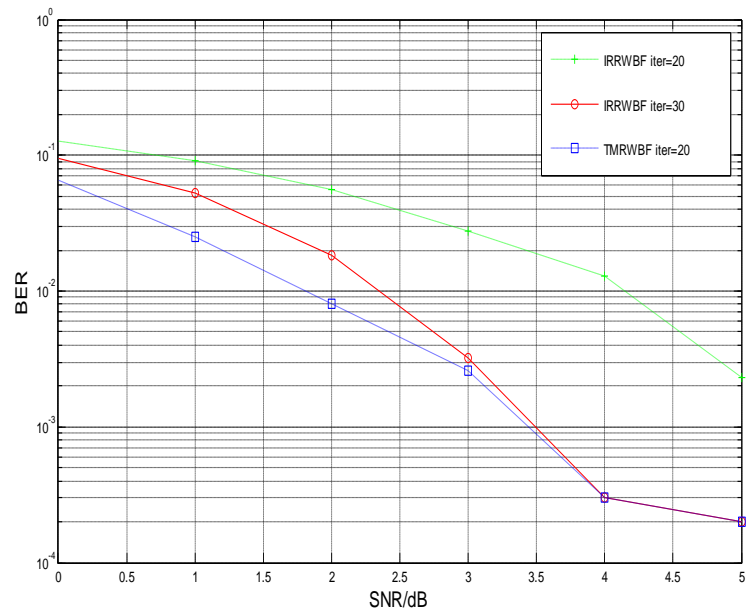

Fig. 5. RRWBF compared with different iterations required in MTRWBF.

In the low SNR range, make a comparison between MTRWBF algorithm and IRRWBF algorithm performance in different number of iterations. Simulation results indicate that the proposed algorithm can use fewer iterations to obtain better decoding performance, which can reduce the iterative decoding frequency as well as time complexity.

\section{CONCLUSIONS}

The proposed MTRWBF algorithm receives bit sequence based on reliability rate and threshold flipped multiple errors in each iteration. With a slight increasing in computing complexity, this algorithm overcomes WBF and numerous variants of WBF algorithm which only considers flipping greater weight and ignores the decoding drawback influenced by the absolute value of symbol. In addition, MTRWBF flips multiple bits each time, which leads 
convergence speed more rapidly than that in IRRWBF decoding algorithm. Simulation results indicates that the proposed algorithm requires less iterations than IRRWBF algorithm and achieves better BER performance.

\section{REFERENCES}

[1] R. G. Gallager, "Low-density parity-check codes," IRE Trans. Information Theory, vol. 8, no. 1, pp. 21-28, Jan. 1962.

[2] Y. Kou, S. Lin, and M. Fossorier, "Low density parity check codes based on finite geometries: A rediscovery and more," IEEE Trans. Inf. Theory, vol. 47, no. 7, pp. 2711-2736, Nov. 2001.

[3] J. Cho, J. Kim, and W. Sung. "VLSI implementation of a highthroughput soft-bit-flipping decoder for geometric LDPC codes," IEEE Trans. on Circuits and Systems, vol. 8, no. 5, pp. 1083-1094, May 2010.

[4] T. Richardson and R. Urbanke, "The capacity of low-density parity check codes under message-passing decoding," IEEE Trans. Inf. Theory, vol. 47, no. 2, pp. 599-618, Feb. 2001.

[5] H. Sankar and K. R. Narayanan, "Memory-efficient sum-product decoding of LDPC codes," IEEE Trans. Commun., vol. 52, no. 8, pp. 1225-1230, Aug. 2004.

[6] C.-L. Wang, X. Chen, Z. Li, and S. Yang, "A simplified min-sum decoding algorithm for non-binary LDPC codes," IEEE Trans. Commun., vol. 61, no. 1, pp. 24-32, Jan. 2013.

[7] J. Heo, "Analysis of scaling soft information on low density parity check codes," IEEE Electron. Lett., vol. 39, no. 2, pp. 219-221, Jan. 2003.

[8] J. Li and X. Zhang, "Hybrid iterative decoding for low-density paritycheck codes based on finite Geometries," IEEE Commun. Lett., vol. 12, no. 9, pp. 29-31, Jan. 2008.

[9] J. Zhang and M. P. C. Fossorier, "A modified weighted bit-flipping decoding of low-density parity-check codes," IEEE Commun. Lett., vol. 8, no. 3, pp. 165-167, Mar. 2004.

[10] F. Guo and L. Hanzo, "Reliability ratio based weighted bit-flipping decoding for low-density parity-check codes," Electron. Lett., vol. 40 , no. 21, pp. 1356-1358, Oct. 2004.

[11] C.-H. Lee and W. Wolf, "Implementation-efficient reliability ratio based weighted bit-flipping decoding for LDPC codes," Electron. Lett., vol. 41, no. 13, pp. 755-757, June 2005.

[12] T. Wadayama, K. Nakamura, M. Yagita, Y. Funahashi, S. Usami, and I. Takumi, "Gradient descent bit flipping algorithms for decoding ldpc codes," IEEE Trans. Commun., vol. 58, no. 6, pp. 1610-1614, June 2010 .
[13] N. Ngatched, F. Takawira, and M. Bossert, "An improved decoding algorithm for finite-geometry LDPC codes," IEEE Trans. Commun., vol. 57, no. 2, pp. 302-306, Feb. 2009.

[14] T.-C. Chen, "Channel-independent weighted bit-flipping decoding algorithm for low-density parity-check codes," IET Commun., vol. 6, no. 17 , pp. $2968-8268$, Nov. 2012.

[15] J.-H. Hung and S.-G. Chen, "An effective multibit-flipping algorithm for LDPC decoding," in Proc. 2nd Int. Conf. Signal Processing and Communication Systems(ICSPCS), Gold Coast, QLD, Dec. 2008, pp. $1-5$.

[16] M. Jiang, C. Zhao, Z. Shi, and Y. Chen, "An improvement on the modified weighted bit flipping decoding algorithm for LDPC codes," IEEE Commun. Lett., vol. 9, no. 9, pp. 814-816, Sep. 2005.

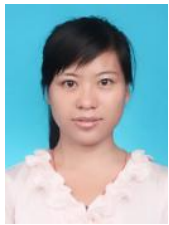

Lina Zhang was born in Shaanxi, China in 1986. She received the B.S. in electronic and information engineering from $\mathrm{Xi}$ 'an University of Science and Technology, Xi'an, China in 2011. She is currently pursuing the master degree at the School of Electronic Science and Engineering, Nanjing University, Nanjing, China. Her research interests include LDPC decoding

algorithms.

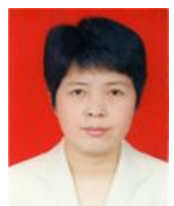

Zhihui Ye was born in Jiangsu, China in 1967. She received the B.S. and Ph.D. degrees from the PLA University of Science and Technology and Southeast University, China in 1988 and 2004, respectively. She is currently a professor in School of Electronic Science and Engineering, Nanjing University. Her research interests include mobile communications and satellite communication.

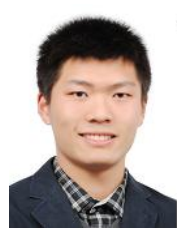

Qi Feng was born in Jiangsu, China, in 1988. He received the B.S. in communications engineering from Nanjing University of Posts and Telecommunications, Nanjing, China, in 2010. He is currently pursuing the $\mathrm{Ph} . \mathrm{D}$. degree at the School of Electronic Science and Engineering, Nanjing University, Nanjing, China. His research interests include wireless communications and satellite communications. 